\title{
Properties of Solutions to Stochastic Set Differential Equations under Non-Lipschitzian Coefficients
}

\author{
Weiyin Fei, Yunhe Li, and Chen Fei \\ School of Mathematics and Physics, Anhui Polytechnic University, Wuhu, Anhui 241000, China \\ Correspondence should be addressed to Weiyin Fei; wyfei@dhu.edu.cn
}

Received 31 October 2013; Revised 7 January 2014; Accepted 19 January 2014; Published 5 March 2014

Academic Editor: Ilaria Fragala

Copyright (C) 2014 Weiyin Fei et al. This is an open access article distributed under the Creative Commons Attribution License, which permits unrestricted use, distribution, and reproduction in any medium, provided the original work is properly cited.

A class of stochastic set differential equations (SSDEs) with non-Lipschitzian coefficients is investigated. We first give the preliminaries on the stochastic set differential equations. Then the nonexplosion of solutions to the SSDEs is discussed. Moreover, the existence and uniqueness of the solutions to SSDEs are proven. Finally, the continuous dependence of the solutions to SSDEs is studied.

\section{Introduction}

Set-valued differential equations which were started in 1969 by de Blasi and Lervolino [1] have been employed in investigations of dynamic systems. The evidence of set differential equations for such areas as control theory, differential inclusions, and fuzzy differential equations can be found in [2-12] and references therein. The set differential equations also are explored in [13-15]. One of the main advantages of investigating deterministic set differential equations is that they can be used as a tool for studying properties of solutions of differential inclusions. On the other hand, the set-valued random processes are first introduced by van Cutsem [16]. Since then the subject has attracted the interest of many mathematicians and further contributions are made from both the theoretical and applied viewpoints (see, e.g., $[17-26]$ ). In [27-31], the set-valued random differential equations are studied. The strong solution of Itô type set-valued stochastic differential equation is analyzed in [32].

As far as we know, there exists a wide literature where attempts have been made to investigate stochastic differential inclusions (see, e.g., [33-40] and references therein). And recently, in [27], a kind of the SSDEs disturbed by Wiener processes is investigated, where under the Lipschitzian condition the existence and uniqueness of solutions to the SSDEs are proven. Under the non-Lipschitzian condition, the existence and uniqueness of solutions to the stochastic set differential equations are proven in $[41,42]$. Moreover, in our present paper, under the non-Lipschitzian condition the nonexplosion and continuous dependence of solutions to the SSDEs are studied. The mathematical tool employed in the paper is the Bihari inequality and the notion of the support function. The work presented here generalizes results obtained both for deterministic and for random set differential equations. Also, it should be noted that the work related to this paper is the discussions of fuzzy-valued processes and stochastic differential equations (see, e.g., [43-51]).

The paper is organized as follows. Section 2 gives an appropriate framework on a set-valued analysis within which the notion of a set-valued stochastic integral is given. In Section 3, moreover, the continuous dependence of the solutions for SSDEs on initial conditions and nonexplosion are discussed. Finally, the conclusions are made in Section 4.

\section{Preliminaries}

Let $\mathscr{K}\left(\mathbf{R}^{d}\right)$ be the family of all nonempty compact and convex subsets of $\mathbf{R}^{d}$. In $\mathscr{K}\left(\mathbf{R}^{d}\right)$, we define the Hausdorff metric $d_{H}$ of two sets $A, B \in \mathscr{K}\left(\mathbf{R}^{d}\right)$ as follows:

$$
d_{H}=\max \left(\sup _{a \in A} \inf _{b \in B}\|a-b\|, \sup _{b \in B} \inf _{a \in A}\|a-b\|\right) .
$$

Throughout this paper, let $(\Omega, \mathscr{A}, P)$ be complete probability space. $\mathscr{A} \times \mathscr{B}_{+}$is a product $\sigma$-field of $\Omega \times$ $\mathbf{R}_{+} . \mathscr{M}\left(\Omega, \mathscr{A} ; \mathscr{K}\left(\mathbf{R}^{d}\right)\right)$ denotes the family of $\mathscr{A}$-measurable 
multifunctions with values in $\mathbf{R}^{d}$. A multifunction $F \in$ $\mathscr{M}\left(\Omega, \mathscr{A} ; \mathscr{K}\left(\mathbf{R}^{d}\right)\right)$ is said to be $L^{p}$-integrably bounded, $p \geq 1$, if there exists $h \in L^{p}\left(\Omega, \mathscr{A}, P ; \mathbf{R}_{+}\right)$such that $|\|F\|| \leq h$ a.s., where

$$
|\|A\||:=d_{H}(A,\{0\})=\sup _{a \in A}\|a\| \quad \text { for } A \in \mathscr{K}\left(\mathbf{R}^{d}\right) .
$$

Let us denote

$$
\begin{aligned}
\mathscr{L}^{p} & \left(\Omega, \mathscr{A}, P ; \mathscr{K}\left(\mathbf{R}^{d}\right)\right) \\
& :=\left\{F \in \mathscr{M}\left(\Omega, \mathscr{A} ; \mathscr{K}\left(\mathbf{R}^{d}\right)\right):|\|F\|| \in L^{p}\left(\Omega, \mathscr{A}, P ; \mathbf{R}_{+}\right)\right\} .
\end{aligned}
$$

Denote $I:=[0, \infty)$. Let $\left(\Omega, \mathscr{A},\left\{\mathscr{A}_{t}\right\}_{t \in I}, P\right)$ be a complete, filtered probability space where the sub- $\sigma$-field family $\left(\mathscr{A}_{t}, t \in\right.$ I) of $\mathscr{A}$ satisfies the usual conditions. We call $X: I \times$ $\Omega \rightarrow \mathscr{K}\left(\mathbf{R}^{d}\right)$ a set-valued stochastic process, if for every $t \in I$ a mapping $X(t, \cdot)=X(t): \Omega \rightarrow \mathscr{K}\left(\mathbf{R}^{d}\right)$ is a set-valued random variable. If $X: I \times \Omega \rightarrow \mathscr{K}\left(\mathbf{R}^{d}\right)$ is $\left\{\mathscr{A}_{t}\right\}_{t \in I}$-adapted and measurable, then it will be called nonanticipating. Equivalently, the set-valued process $X$ is nonanticipating if and only if $X$ is measurable with respect to the $\sigma$-algebra $\mathcal{N}$, which is defined as follows:

$$
\mathscr{N}:=\left\{A \in \mathscr{B}(I) \otimes \mathscr{A}: A^{t} \in \mathscr{A}_{t} \text { for every } t \in I\right\},
$$

where $A^{t}=\{\omega:(t, \omega) \in A\}$ for $t \in I$.

Let $p \geq 1$ and $L^{p}\left(I \times \Omega, \mathscr{N} ; \mathbf{R}^{d}\right)$ denote the set of all nonanticipating $\mathbf{R}^{d}$-valued stochastic processes $\{h(t)\}_{t \in I}$ such that $E\left(\int_{0}^{T}\|h(s)\|^{p} d s\right)<\infty$. A set-valued stochastic process $X$ is called $L^{p}$-integrably bounded, if there exists a real-valued stochastic process $\hbar \in L^{p}\left(I \times \Omega, \mathcal{N} ; \mathbf{R}_{+}\right)$such that

$$
\||X(t, \omega)|\| \leq \hbar(t, \omega) \quad \text { for a.a. }(t, \omega) \in I \times \Omega \text {. }
$$

We define the operation on $\mathscr{K}\left(\mathbf{R}^{d}\right)$ as follows. For two sets $A, B \in \mathscr{K}\left(\mathbf{R}^{d}\right)$, if there exists such a $C \in \mathscr{K}\left(\mathbf{R}^{d}\right)$ that $A=$ $B+C$, then $C$ is the Hukuhara difference of $A$ and $B$ denoted by $C=A \ominus B$. We note that $A+B=\{0\}$ implies that $A=-B$. However, $A \ominus A \neq\{0\}$. Indeed, take $A=[0,1]$.

It is well known that

$$
\begin{aligned}
& d_{H}(A+C, B+C)=d_{H}(A, B), \\
& d_{H}(\lambda A, \lambda B)=|\lambda| d_{H}(A, B),
\end{aligned}
$$

for all $A, B, C \in \mathscr{K}\left(\mathbf{R}^{d}\right), \lambda \in \mathbf{R}$.

Let $\sigma(A, r) \triangleq \sup \{\langle x, r\rangle: x \in A\} ; r \in \mathbf{B}$ is the support function of $A$, where $\mathbf{B}$ is a unit sphere centered at origin. The support function $\sigma(\cdot, \cdot)$ satisfies the following properties.

(i) $\sigma(A, \cdot)$ is bounded on $\mathbf{B}$; that is, $|\sigma(A, r)| \leq|\|A\||, \forall r \in$ B.

(ii) $\sigma(A, \cdot)$ is Lipschitz continuous in $r$

$$
\left|\sigma(A, r)-\sigma\left(A, r^{*}\right)\right| \leq|\|A\|| \cdot\left|r-r^{*}\right|, \quad \forall r, r^{*} \in \mathbf{B} .
$$

(iii) For all $A, B \in \mathscr{K}\left(\mathbf{R}^{d}\right)$,

$$
H(A, B)=\sup _{r \in \mathbf{B}}|\sigma(A, r)-\sigma(B, r)| .
$$

(iv) For all $A, B \in \mathscr{K}\left(\mathbf{R}^{d}\right), \sigma(A+B, r)=\sigma(A, r)+$ $\sigma(B, r) \forall r \in \mathbf{B}$.

(v) For all $A \in \mathscr{K}\left(\mathbf{R}^{d}\right), \sigma(\lambda A, r)=\lambda \sigma(A, r) \forall r \in \mathbf{B}, \lambda \geq$ 0 .

(vi) For all $A, B \in \mathscr{K}\left(\mathbf{R}^{d}\right)$, if $\sigma(A, r)=\sigma(A, r)+$ $\sigma(B, r), \forall r \in \mathbf{B}$; then we have $A=B$.

By $\mathscr{L}^{p}\left(I \times \Omega, \mathscr{N} ; \mathscr{K}\left(\mathbf{R}^{d}\right)\right)$ we denote the set of nonanticipating and $L^{p}$-integrably bounded set-valued stochastic processes. Let $X \in \mathscr{L}^{1}\left(I \times \Omega, \mathscr{N} ; \mathscr{K}\left(\mathbf{R}^{d}\right)\right)$. For such $X$ and a fixed $t \in I$, by the Fubini Theorem, we can define Aumann's integral $\int_{0}^{t} X(s, \omega) d s$, for $\omega \in \Omega$. Obviously, for every $t \in I$ and $\omega \in \Omega$ the Aumann integral $\int_{0}^{t} X(s, \omega) d s$ belongs to $\mathscr{K}\left(\mathbf{R}^{d}\right)$ (see, e.g., $[7,19]$ ).

We say that a set-valued stochastic process $X$ is $d_{H^{-}}$ continuous, if almost all its trajectories, that is, the mappings $X(\cdot, \omega): I \rightarrow \mathscr{K}\left(\mathbf{R}^{d}\right)$, are $d_{H}$-continuous functions. It is easy to know that if $X \in \mathscr{L}^{p}\left(I \times \Omega, \mathscr{N} ; \mathscr{K}\left(\mathbf{R}^{d}\right)\right)$, then the setvalued stochastic process $\int_{0}^{t} X(s) d s$ is $d_{H^{-}}$-continuous (see, e.g., Corollary 1 in [27]).

In what follows, we state the generalized Bihari inequality (cf., Mao [52]) which plays an important role in the following section.

Lemma 1 (generalized Bihari inequality). Let $u$ be Borel measurable, bounded, nonnegative, and left limit function on $[0, T]$ and $c>0$. Let $K: \mathbf{R}_{+} \rightarrow \mathbf{R}_{+}$be a continuous nondecreasing function such that $K(t)>0$ for all $t>0$.

(i) If $\mu(t)$ is a continuous nonnegative nondecreasing function on $[0, T]$, then the inequality

$$
u(t) \leq c+\int_{0}^{t} K\left(u\left(s^{-}\right)\right) d \mu(s), \quad \forall t \in[0, T]
$$

implies that

$$
u(t) \leq G^{-1}(G(c)+\mu(t)),
$$

for all $t \in[0, T]$ such that

$$
G(c)+\mu(t) \in \operatorname{Dom}\left(G^{-1}\right),
$$

where

$$
G(q)=\int_{a}^{q} \frac{1}{K(v)} d v, \quad q>0
$$

where $G^{-1}$ is the inverse function of $G$ and $a \in[0, T]$.

(ii) If $v(t)$ is a continuous nonpositive nonincreasing function on $[0, T]$, then the inequality

$$
u(t) \geq c+\int_{0}^{t} K(u(s-)) d v(s), \quad \forall t \in[0, T],
$$


implies that

$$
u(t) \geq G^{-1}(G(c)+v(t))
$$

for all $t \in[0, T]$ such that

$$
G(c)+v(t) \in \operatorname{Dom}\left(G^{-1}\right) .
$$

\section{Properties of Solutions to SSDEs}

In this section, we consider the following stochastic set differential equation (in the integral form):

$$
X(t)=x_{0}+\int_{0}^{t} f(X(s)) d s+\int_{0}^{t} g(X(s)) d W(s)
$$

where $f$ takes values in $C\left(\mathscr{K}\left(\mathbf{R}^{d}\right), \mathscr{K}\left(\mathbf{R}^{d}\right)\right), \quad g$ in $C\left(\mathscr{K}\left(\mathbf{R}^{d}\right), \mathbf{R}^{d} \times \mathbf{R}^{m}\right), W=(W(t), t \geq 0)$ is an $m$-dimensional Brownian motion, and $x_{0} \in \mathscr{K}\left(\mathbf{R}^{d}\right)$ is a set-valued random variable. Here, $C\left(\mathscr{K}\left(\mathbf{R}^{d}\right), \mathscr{K}\left(\mathbf{R}^{d}\right)\right)$ (resp., $C\left(\mathscr{K}\left(\mathbf{R}^{d}\right), \mathbf{R}^{d} \times \mathbf{R}^{m}\right)$ stands for the family of the continuous functions from the space $\mathscr{K}\left(\mathbf{R}^{d}\right)$ to the space $\mathscr{K}\left(\mathbf{R}^{d}\right)$ (resp., $\mathscr{K}\left(\mathbf{R}^{d}\right)$ to $\left.\mathbf{R}^{d} \times \mathbf{R}^{m}\right)$. In (16), the integral $\int_{0}^{t} f(X(s)) d s$ is Aumann's one, and the integral $\int_{0}^{t} g(X(s)) d W(s)$ is a general Itô type stochastic one, whose definition can refer to [53].

Due to the continuity of $f$, we can know that $\sigma(f(\cdot), r)$ takes values in $C\left(\mathscr{K}\left(\mathbf{R}^{d}\right), \mathbf{R}\right)$. In order to discuss the solutions to (16), by the concept and properties of the support function we consider the following single valued stochastic differential equation, for $\forall r \in \mathbf{B}$ :

$$
\begin{aligned}
d \sigma(X(t), r) & =\sigma(f(X(t)), r) d t+r^{\top} g(X(t)) d W(t), \\
\sigma(X(0), r) & =\sigma\left(x_{0}, r\right) .
\end{aligned}
$$

We first claim that (16) is equivalent to (17). Indeed, from the properties of the support function and (16) we deduce that, for $r \in \mathbf{B}$,

$$
\begin{aligned}
\sigma(X(t), r)= & \sigma\left(x_{0}, r\right)+\sigma\left(\int_{0}^{t} f(X(t)) d t, r\right) \\
& +\int_{0}^{t} r^{\top} g(X(t)) d W(t) \\
= & \sigma\left(x_{0}, r\right)+\int_{0}^{t} \sigma(f(X(t)), r) d t \\
& +\int_{0}^{t} r^{\top} g(X(t)) d W(t),
\end{aligned}
$$

which shows that (17) holds. Conversely, (16) can be derived from (17).

It is known that (17) has a solution up to a lifetime $\zeta(r)$ which depends on $r \in \mathbf{B}$. Set

$$
\zeta=\inf _{r \in \mathbf{B}} \zeta(r)
$$

We call $\zeta$ the lifetime of solution to (16). Obviously, $\||X(\zeta)|\|=$ $+\infty$ a.s. Here, by the concepts of explosion time and lifetime time from Pages 158 and 191 in [54], the lifetime of solution to (16) or (17) is the same as the explosion time of the solution of (16) or (17).

If (17) has the pathwise uniqueness, then we show the existence and uniqueness of the solution to the SSDE (16). So the study of pathwise uniqueness is of great interest. It is a classical result that, under the Lipschitz coefficients, the pathwise uniqueness holds and the solution of (17) can be constructed by using Picard iteration; moreover, the solution depends on the initial values continuously. However, under non-Lipschitzian condition, Fei [42] presents the existence and uniqueness of solutions to (16); hence, the existence and uniqueness of solutions to (17) also are proven.

In what follows, we discuss the nonexplosion of the solutions to (16) under non-Lipschitzian condition. Our idea is to derive an inequality so that the generalized Bihari inequality (Lemma 1) can be applied.

Theorem 2. Let $\rho: \mathbf{R}_{+} \rightarrow[1,+\infty)$ be a continuous function such that

(i) $s \rho(s)$ is nondecreasing and concave;

(ii) $\int_{0}^{\infty} d s /(s \rho(s)+1)=+\infty$.

Assume that, for some constant $C>0$,

$$
\begin{aligned}
\|f(x) \mid\| & \leq C\left(\||x|\| \rho\left(\||x|\|^{2}\right)+1\right), \\
\|g(x)\|^{2} & \leq C\left(\||x|\|^{2} \rho\left(\||x|\|^{2}\right)+1\right), \quad x \in \mathscr{K}\left(\mathbf{R}^{d}\right) .
\end{aligned}
$$

If $E\left\|\left|x_{0}\right|\right\|<+\infty$, then the lifetime of the solution $X\left(t, x_{0}\right)$ to (16) is infinite: $\zeta=+\infty$ a.s. Moreover,

$$
\lim _{E\left\|\left|x_{0}\right|\right\| \rightarrow+\infty} E\left\|\left|X\left(t, x_{0}\right)\right|\right\|=+\infty \quad \forall t \geq 0 .
$$

Proof. Let $\xi^{r}(t)=|\sigma(X(t), r)|^{2}, \forall r \in \mathbf{B}$, where $X(t)$ is a solution to (16). Hence, we have

$$
\begin{aligned}
d \xi^{r}(t)= & {\left[2 \sigma(X(t), r) \sigma(f(X(t)), r)+\left\|g(X(t))^{\top} r\right\|^{2}\right] d t } \\
& +2 \sigma(X(t), r) r^{\top} g(X(t)) d W(t) .
\end{aligned}
$$

Since $r \in \mathbf{B}$, we get $\left\|g(X(s))^{\top} r\right\|^{2} \leq\|g(X(s))\|^{2}$. Set $\xi(t)=$ $\||X(t)|\|^{2}$; it follows that from (20) and property (i) of the support function $\sigma(\cdot)$

$$
\begin{aligned}
E \xi^{r}(t)=\xi^{r}(0)+2 E \int_{0}^{t}(\sigma & (X(s), r) \sigma(f(X(s)), r) \\
+ & \left.\left\|r^{\top} g(X(s))\right\|^{2}\right) d s
\end{aligned}
$$




$$
\begin{array}{r}
\leq E\left\|\left|x_{0}\right|\right\|^{2}+2 C \int_{0}^{t} E(2 \xi(s) \rho(\xi(s)) \\
+\sqrt{\xi(s)}+1) d s \\
\leq E\left\|\left|x_{0}\right|\right\|^{2}+2 C \int_{0}^{t}(2 E \xi(s) \rho(E \xi(s)) \\
+\sqrt{E \xi(s)}+1) d s,
\end{array}
$$

where we have utilized the concavity of the functions $v \rho(v)$ and $\sqrt{v}$.

Denote $\eta(t)=E \xi(t)$. Noticing $\rho(\eta) \geq 1$, we get

$$
\frac{\eta \rho(\eta)+\sqrt{\eta}}{\eta \rho(\eta)+1} \leq 1+\frac{\sqrt{\eta}}{\eta \rho(\eta)+1} \leq 1+\frac{\sqrt{\eta}}{\eta+1} \leq 1+\frac{1}{2}<2,
$$

where $\varphi(\eta)=\sqrt{\eta} /(1+\eta)$ takes the maximum at 1 on the interval $(0, \infty)$.

It is easy to see that

$$
\sup _{\eta \geq 0} \frac{\eta \rho(\eta)+\sqrt{\eta}}{\eta \rho(\eta)+1} \leq 2
$$

which deduce that

$$
\frac{2 \eta \rho(\eta)+\sqrt{\eta}}{\eta \rho(\eta)+1} \leq \frac{2(\eta \rho(\eta)+\sqrt{\eta})}{\eta \rho(\eta)+1}+\frac{1}{\eta \rho(\eta)+1} \leq 5 .
$$

Thus we have

$$
2 \eta \rho(\eta)+\sqrt{\eta}+1 \leq 6(\eta \rho(\eta)+1) .
$$

In virtue of (23), we have

$$
\eta(t) \leq E\left\|\left|x_{0}\right|\right\|^{2}+12 C \int_{0}^{t}(\eta(s) \rho(\eta(s))+1) d s .
$$

Set

$$
G(u)=\int_{1}^{u} \frac{d s}{s \rho(s)+1}, \quad u>0 .
$$

By condition (ii) it is easy to show that $G(u)$ is strictly increasing, $G(u) \rightarrow+\infty$ as $u \rightarrow+\infty$ and $G^{-1}(u) \rightarrow+\infty$ as $u \rightarrow+\infty$.

From (28), the generalized Bihari inequality (Lemma 1 (i)), and $E\left\|\left|x_{0}\right|\right\|<+\infty$ we obtain

$$
\eta(t) \leq G^{-1}\left(G\left(E\left\|\left|x_{0}\right|\right\|^{2}\right)+12 C t\right)<+\infty, \quad \forall t \geq 0,
$$

which proves that $\zeta=+\infty$.
On the other hand, from (20) and property (i) of the support function $\sigma(\cdot)$ we have

$$
\begin{aligned}
& E \xi^{r}(t)= \xi^{r}(0) \\
&+2 E \int_{0}^{t}(\sigma(X(s), r) \sigma(f(X(s)), r) \\
&\left.+\left\|r^{\top} g(X(s))\right\|^{2}\right) d s \\
& \geq E\left\|x_{0} \mid\right\|^{2}-2 C \int_{0}^{t} E(\xi(s) \rho(\xi(s))+\sqrt{\xi(s)}) d s \\
& \geq E\left\|x_{0} \mid\right\|^{2}-2 C \int_{0}^{t}(E \xi(s) \rho(E \xi(s))+\sqrt{E \xi(s)}) d s .
\end{aligned}
$$

Due to inequality (24), we have

$$
\eta(t) \geq E\left\|\left|x_{0}\right|\right\|^{2}-4 C \int_{0}^{t}(\eta(s) \rho(\eta(s))+1) d s .
$$

By Lemma 1(ii), we get

$$
E\left\|\left|X\left(t, x_{0}\right)\right|\right\|^{2}=\eta(t) \geq G^{-1}\left(G\left(E\left\|\left|x_{0}\right|\right\|^{2}\right)-4 C t\right), \quad \forall t \geq 0,
$$

which shows that $\lim _{E\left\|\left|x_{0}\right|\right\| \rightarrow+\infty} E\left\|\left|X\left(t, x_{0}\right)\right|\right\|=+\infty$ by the property of the function $G(\cdot)$ in (29). Thus, the proof of the theorem is complete.

Theorem 3. Let $\psi:(0,1) \rightarrow[1,+\infty)$ be a continuous function such that

(i) $s \psi(s)$ is nondecreasing and concave;

(ii) $\int_{0}^{1 / 2} d s / s \psi(s)=+\infty$.

Assume that for some constant $C>0, d_{H}(x, y)<$ $1, x, y \in \mathscr{K}\left(\mathbf{R}^{d}\right), t \in I$,

$$
\begin{gathered}
d_{H}(f(t, x), f(t, y)) \leq C d_{H}(x, y) \psi\left(d_{H}^{2}(x, y)\right), \\
\| g(t, x)-g(t, y)) \|^{2} \leq C d_{H}^{2}(x, y) \psi\left(d_{H}^{2}(x, y)\right) .
\end{gathered}
$$

Then SSDE (16) has a unique solution.

Proof. By constructing the sequence $\left\{X_{n}\right\}$ of set-valued random variables as that in [42], the existence of the solutions to (16) is similarly proven. Next, we prove the uniqueness of the solutions to (16).

Let $(X(t))_{t \geq 0}$ and $(Y(t))_{t \geq 0}$ be two solutions of (16). Set $\eta^{r}(t)=\sigma(X(t), r)-\sigma(Y(t), r), \xi^{r}(t)=\left|\eta^{r}(t)\right|^{2}, \forall r \in \mathbf{B}$. Hence, by Itô formula we have

$$
\begin{aligned}
d \xi^{r}(t)=[ & 2 \eta^{r}(t)(\sigma(f(t, X(t)), r)-\sigma(f(t, Y(t)), r)) \\
& \left.+\left\|(g(t, X(t))-g(t, Y(t)))^{\top} r\right\|^{2}\right] d t \\
& +2 \eta^{r}(t) r^{\top}(g(t, X(t))-g(t, Y(t))) d W(t) .
\end{aligned}
$$


Since $r \in \mathbf{B}$, we get $\left\|(g(t, X(s))-g(t, Y(s)))^{\top} r\right\|^{2} \leq$ $\|g(t, X(s))-g(t, Y(s))\|^{2}$. Set $\xi(t)=d_{H}^{2}(X(t), Y(t))$.

From condition (34) and the property of the support function, we get

$$
\begin{aligned}
& \left|\eta^{r}(t)\right| \leq d_{H}(X(t), Y(t)), \\
& |\sigma(f(t, X(t)), r)-\sigma(f(t, Y(t)), r)| \\
& \quad \leq d_{H}(f(t, X(t)), f(t, Y(t))) \\
& \quad \leq C d_{H}(X(t), Y(t)) \psi\left(d_{H}^{2}(X(t), Y(t))\right),
\end{aligned}
$$

which deduces

$$
\begin{aligned}
& E \xi^{r}(t) \\
& =2 E \int_{0}^{t}\left(\eta^{r}(s)(\sigma(f(t, X(s)), r)-\sigma(f(t, Y(s)), r))\right. \\
& \left.+\left\|(g(t, X(s))-g(t, Y(s)))^{\top} r\right\|^{2}\right) d s \\
& \leq 2 C \int_{0}^{t} E\left(2 d_{H}^{2}(X(s), Y(s)) \psi\right. \\
& \left.\times\left(d_{H}^{2}(X(s), Y(s))\right)\right) d s \\
& \leq 4 C \int_{0}^{t} E \xi(s) \psi(E \xi(s)) d s,
\end{aligned}
$$

where we have utilized the concavity of the functions $v \psi(v)$.

Denote $\vartheta(t)=E \xi(t)$. In virtue of (37), we have

$$
\vartheta(t) \leq 4 C \int_{0}^{t} \vartheta(s) \psi(\vartheta(s)) d s .
$$

Let

$$
G(u)=\int_{1 / 2}^{u} \frac{d s}{s \psi(s)}, \quad u>0 .
$$

We easily show that $G(u)$ is strictly increasing, $G(u) \rightarrow-\infty$ as $u \rightarrow 0$ and $G^{-1}(u) \rightarrow 0$ as $u \rightarrow-\infty$.

By Lemma 1 (i), we obtain

$$
\vartheta(t) \leq G^{-1}(G(0+)+4 C t)=0, \quad \forall t \geq 0,
$$

which shows that $X(t)=Y(t)$. Thus we complete the proof.

Note that function

$$
\psi(s)= \begin{cases}\log \frac{1}{s}, & 0<s \leq \frac{1}{e}, \\ 1, & s>\frac{1}{e},\end{cases}
$$

is a typical example satisfying conditions (i) and (ii).

Next, we will study the dependence of the solutions to the $\operatorname{SSDE}(16)$ on initial data. For the mapping $x_{0} \rightarrow X\left(t, x_{0}\right)$, we call $X_{t}\left(x_{0}\right)=X\left(t, x_{0}\right)$ mean square continuous on $x_{0}$, uniformly with respect to $t \in I$ if $E d_{H}\left(X\left(t, y_{0}\right), X\left(t, x_{0}\right)\right) \rightarrow$ 0 as $d_{H}-\lim y_{0}=x_{0}$ on any compact subset $I$ of $t$, where the limit $x_{0}$ of $y_{0}$ is in sense of the metric $d_{H}$.
Theorem 4. Assume that the conditions in Theorem 3 hold. Then the mapping $x_{0} \rightarrow X_{t}\left(x_{0}\right)$ is mean square continuous, uniformly with respect to $t$ in any compact subset, where $X_{t}\left(x_{0}\right)=X\left(t, x_{0}\right)$ is the solution to SSDE (16).

Proof. Take $\epsilon \in(0,1)$. Consider a small parameter $0<\delta<\epsilon$. Assume $x_{0}, y_{0} \in \mathscr{K}\left(\mathbf{R}^{d}\right)$ such that $d_{H}\left(x_{0}, y_{0}\right)<\delta$. For $\forall r \in \mathbf{B}$, let

$$
\begin{gathered}
\eta^{r}(t)=\sigma\left(X_{t}\left(x_{0}\right), r\right)-\sigma\left(X_{t}\left(y_{0}\right), r\right), \\
\xi^{r}(t)=\left|\eta^{r}(t)\right|^{2}, \\
\xi(t)=d_{H}^{2}\left(X_{t}\left(x_{0}\right), X_{t}\left(y_{0}\right)\right) .
\end{gathered}
$$

By (17) and Itô formula, we have

$d \xi^{r}(t)$

$$
\begin{gathered}
=\left[2 \eta ^ { \alpha , r } ( t ) \left(\sigma\left(f\left(t, X\left(t, x_{0}\right)\right), \alpha, r\right)\right.\right. \\
\left.-\sigma\left(f\left(t, X\left(t, y_{0}\right)\right), r\right)\right) \\
\left.+\left\|\left(g\left(t, X\left(t, x_{0}\right)\right)-g\left(t, X\left(t, y_{0}\right)\right)\right)^{\top} r\right\|^{2}\right] d t \\
+2 \eta^{\alpha, r}(t) r^{\top}\left(g\left(t, X\left(t, x_{0}\right)\right)\right. \\
\left.-g\left(t, X\left(t, y_{0}\right)\right)\right) d W(t) .
\end{gathered}
$$

From condition (34) and the property of the support function, we get

$$
\begin{aligned}
& \left|\sigma\left(f\left(t, X\left(t, x_{0}\right)\right), r\right)-\sigma\left(f\left(t, X\left(t, y_{0}\right)\right), r\right)\right| \\
& \leq d_{H}\left(f\left(t, X\left(t, x_{0}\right)\right), f\left(t, X\left(t, y_{0}\right)\right)\right) \\
& \leq C d_{H}\left(X\left(t, x_{0}\right), X\left(t, y_{0}\right)\right) \psi\left(d_{H}^{2}\left(X\left(t, x_{0}\right), X\left(t, y_{0}\right)\right)\right),
\end{aligned}
$$

which deduces

$$
\begin{aligned}
& E \xi^{r}(t) \\
& =E \xi^{r}(0) \\
& +2 E \int_{0}^{t}\left(\eta ^ { \alpha , r } ( s ) \left(\sigma\left(f\left(t, X\left(s, x_{0}\right)\right), r\right)\right.\right. \\
& \left.-\sigma\left(f\left(t, X\left(s, y_{0}\right)\right), r\right)\right) \\
& \left.+\left\|\left(g\left(t, X\left(s, x_{0}\right)\right)-g\left(t, X\left(s, y_{0}\right)\right)\right)^{\top} r\right\|^{2}\right) d s \\
& +2 C d_{H}^{2}\left(x_{0}, y_{0}\right) \quad\left(2 d_{H}^{2}\left(X\left(s, x_{0}\right), X\left(s, y_{0}\right)\right) \psi\right. \\
& \left.\times\left(d_{H}^{2}\left(X\left(s, x_{0}\right), X\left(s, y_{0}\right)\right)\right)\right) d s \\
& \leq E d_{H}^{2}\left(x_{0}, y_{0}\right)+4 C \int_{0}^{t} E \xi(s) \psi(E \xi(s)) d s,
\end{aligned}
$$

where we have utilized the concavity of the functions $v \psi(v)$. 
Setting $\vartheta(t)=E d_{H}^{2}\left(X_{t}\left(x_{0}\right), X_{t}\left(y_{0}\right)\right)$, from (45) we have

$$
\vartheta(t) \leq E d_{H}^{2}\left(x_{0}, y_{0}\right)+4 C \int_{0}^{t} \vartheta(s) \psi(\vartheta(s)) d s .
$$

Define

$$
G(u)=\int_{a}^{u} \frac{d s}{s \psi(s)}, \quad u>0, \text { for some } a \in(0,1) .
$$

We easily have that $G(u)$ is strictly increasing, $G(u) \rightarrow-\infty$ as $u \rightarrow 0$ and $G^{-1}(u) \rightarrow 0$ as $u \rightarrow-\infty$.

By Lemma 1 (i), we have

$$
\vartheta(t) \leq G^{-1}\left(G\left(E d_{H}^{2}\left(x_{0}, y_{0}\right)\right)+4 C t\right) .
$$

For arbitrary $\epsilon>0$ and given $t$, it is easy to deduce that there exists $\delta>0$ with $d_{H}\left(x_{0}, y_{0}\right)<\delta$, which shows $E d_{H}\left(x_{0}, y_{0}\right)<$ $\delta$, such that

$$
\vartheta(t) \leq G^{-1}\left(G\left(E d_{H}^{2}\left(x_{0}, y_{0}\right)\right)+4 C t\right)<\epsilon^{2} .
$$

Since $G(u)$ is increasing, we have that

$$
\begin{aligned}
\sup _{0 \leq s \leq t} \vartheta(s) & \leq \sup _{0 \leq s \leq t} G^{-1}\left(G\left(E d_{H}^{2}\left(x_{0}, y_{0}\right)\right)+4 C s\right) \\
& \leq G^{-1}\left(G\left(E d_{H}^{2}\left(x_{0}, y_{0}\right)\right)+4 C t\right)<\epsilon^{2},
\end{aligned}
$$

which shows that

$$
\begin{array}{rl}
\sup _{0 \leq s \leq t} & E d_{H}\left(X_{s}\left(x_{0}\right), X_{s}\left(y_{0}\right)\right) \\
& \leq \sup _{0 \leq s \leq t} \sqrt{E d_{H}^{2}\left(X_{s}\left(x_{0}\right), X_{s}\left(y_{0}\right)\right)}<\epsilon .
\end{array}
$$

Thus, we show $X_{t}\left(x_{0}\right)$ is mean square continuous on $x_{0}$, with respect to $t$ in any compact subset. Therefore, we complete the proof.

Definition 5. The two solutions $X_{t}\left(x_{0}\right)$ and $Y_{t}\left(y_{0}\right)$ of SSDE (16) with initial value $x_{0}$ and $y_{0}$, respectively, if for any $t$, $P\left(d_{H}\left(X_{t}\left(x_{0}\right), X_{t}\left(y_{0}\right)\right)>0\right)>0$, can be called nonconfluence.

The following theorem gives the sufficient condition.

Theorem 6. Suppose that the conditions in Theorem 3 hold. For $\forall x_{0}, y_{0} \in \mathscr{K}\left(\mathbf{R}^{d}\right), x_{0} \neq y_{0}$, we have that $X_{t}\left(x_{0}\right)$ and $X_{t}\left(y_{0}\right)$ are nonconfluence.

Proof. Let

$$
\begin{gathered}
\eta^{r}(t)=\sigma\left(X_{t}\left(x_{0}\right), r\right)-\sigma\left(X_{t}\left(y_{0}\right), r\right), \\
\xi^{r}(t)=\left|\eta^{r}(t)\right|^{2}, \\
\xi(t)=d_{H}^{2}\left(X_{t}\left(x_{0}\right), X_{t}\left(y_{0}\right)\right), \\
\vartheta(t)=E \xi(t) .
\end{gathered}
$$

From condition (34) and the property of the support function, for $\forall r \in \mathbf{B}$, we get

$$
\begin{aligned}
& \left|\sigma\left(f\left(t, X\left(t, x_{0}\right)\right), r\right)-\sigma\left(f\left(t, X\left(t, y_{0}\right)\right), r\right)\right| \\
& \quad \leq d_{H}\left(f\left(t, X\left(t, x_{0}\right)\right), f\left(t, X\left(t, y_{0}\right)\right)\right) \\
& \quad \leq C d_{H}\left(X\left(t, x_{0}\right), X\left(t, y_{0}\right)\right) \psi\left(d_{H}^{2}\left(X\left(t, x_{0}\right), X\left(t, y_{0}\right)\right)\right) .
\end{aligned}
$$

Thus, similar to the discussion in the proof of Theorem 4, we obtain

$$
\begin{aligned}
& E \xi^{r}(t) \\
& =E \xi^{r}(0) \\
& +2 E \int_{0}^{t}\left(\eta ^ { r } ( s ) \left(\sigma\left(f\left(t, X\left(s, x_{0}\right)\right), r\right)\right.\right. \\
& \left.-\sigma\left(f\left(t, X\left(s, y_{0}\right)\right), r\right)\right) \\
& \left.+\left\|\left(g\left(t, X\left(s, x_{0}\right)\right)-g\left(t, X\left(s, y_{0}\right)\right)\right)^{\top} r\right\|^{2}\right) d s \\
& \left.\geq E d_{H}^{2}\left(x_{0}, y_{0}\right) \quad \times\left(d_{H}^{2}\left(X\left(s, x_{0}\right), X\left(s, y_{0}\right)\right)\right)\right) d s \\
& -2 C \int_{0}^{t} E\left(2 d_{H}^{2}\left(X\left(s, x_{0}\right), X\left(s, y_{0}\right)\right) \psi\right. \\
& \geq E d_{H}^{2}\left(x_{0}, y_{0}\right)-4 C \int_{0}^{t} E \xi(s) \psi(E \xi(s)) d s,
\end{aligned}
$$

which shows that

$$
\vartheta(t) \geq E d_{H}^{2}\left(x_{0}, y_{0}\right)-4 C \int_{0}^{t} \vartheta(s) \psi(\vartheta(s)) d s .
$$

Take $G(u)$ as in the proof of Theorem 4.

By Lemma 1 (ii), we have

$$
\vartheta(t) \geq G^{-1}\left(G\left(E d_{H}^{2}\left(x_{0}, y_{0}\right)\right)-4 C t\right), \quad \forall t \geq 0 .
$$

Since $E d_{H}^{2}\left(x_{0}, y_{0}\right) \neq 0$, from the property of $G(u)$, we obtain

$$
\vartheta(t)>0, \quad \forall t \geq 0,
$$

which means that $P\left(d_{H}\left(X_{t}\left(x_{0}\right), X_{t}\left(y_{0}\right)\right)>0\right)>0$. Thus, the proof is complete.

Finally, through using (34), Theorem 6, and the standard arguments, we obtain the following theorem.

Theorem 7. Let condition (34) hold. Then for anyt $>0, x_{0} \rightarrow$ $X_{t}\left(x_{0}\right)$ defines a flow of homeomorphisms of $\mathscr{K}\left(\mathbf{R}^{d}\right)$. 


\section{Conclusions}

In many real dynamic systems, we are often faced with random experiments whose outcomes might be multivalued. Moreover, the stochastic set differential equations may be employed in characterizing a large class of physically important dynamic systems which can be applied in such areas as control, economics, and finance. In this paper, we study the behavior of solutions to SSDEs disturbed by a Wiener process with the non-Lipschitzian coefficients. First, the nonexplosion theorem of the Itô type SSDEs is proven. Then the existence and uniqueness theorem of solutions to SSDEs is given. Moreover, the continuous dependence of solutions to the SSDEs is investigated. Main mathematical tool is the notion of the support function and the generalized Bihari inequality. Besides, the present case can be extended to the SSDEs driven by a multidimensional semimartingale in future.

\section{Conflict of Interests}

The authors declare that there is no conflict of interests regarding the publication of this paper.

\section{Acknowledgments}

The author wishes to express his many thanks to the referees for many valuable suggestions and comments on this paper. This paper is supported by the National Natural Science Foundation of China (71171003; 71271003), Anhui Natural Science Foundation (10040606003), and Anhui Natural Science Foundation of Universities (KJ2012B019, KJ2013B023).

\section{References}

[1] F. S. de Blasi and F. Iervolino, "Equazioni differenziali con soluzioni a valore compatto convesso," Bollettino della Unione Matematica Italiana, vol. 4, no. 2, pp. 194-501, 1969.

[2] F. S. de Blasi, "Banach-Saks-Mazur and Kakutani-Ky Fan theorems in spaces of multifunctions and applications to set differential inclusions," Dynamic Systems and Applications, vol. 16, no. 1, pp. 73-89, 2007.

[3] P. Diamond and P. Kloeden, Metric Spaces of Fuzzy Sets: Theory and Applications, World Scientific Publishing, Singapore, 1994.

[4] W. Y. Fei, "Existence and uniqueness of solution for fuzzy random differential equations with non-Lipschitz coefficients," Information Sciences, vol. 177, no. 20, pp. 4329-4337, 2007.

[5] W. Y. Fei, "Existence and uniqueness for solutions to fuzzy stochastic differential equations driven by local martingales under the non-Lipschitzian condition," Nonlinear Analysis A, vol. 76, pp. 202-214, 2013.

[6] W. Y. Fei, H. J. Liu, and W. Zhang, "On solutions to fuzzy stochastic eqautions with local martingales," Systems and Control Letters, vol. 65, pp. 96-105, 2014.

[7] M. Kisielewicz, Differential Inclusions and Optimal Control, Kluwer Academic Publishers, Dordrecht, The Netherlands, 1991.

[8] V. Lakshmikantham, T. Gnana Bhaskar, and J. Vasundhara Devi, Theory of Set Differential Equations in Metric Spaces, Cambridge Scientific Publishers, Cambridge, UK, 2006.
[9] V. Laksmikantham, S. Leela, and A. S. Vatsala, "Interconnection between set and fuzzy differential equations," Nonlinear Analysis A, vol. 54, no. 2, pp. 351-360, 2003.

[10] V. Lakshmikantham and R. N. Mohapatra, Theory of Fuzzy Differential Equations and Inclusions, Taylor \& Francis, London, UK, 2003.

[11] J. J. Nieto and R. Rodríguez-López, "Bounded solutions for fuzzy differential and integral equations," Chaos, Solitons and Fractals, vol. 27, no. 5, pp. 1376-1386, 2006.

[12] A. Tolstonogov, Differential Inclusions in a Banach Space, Kluwer Academic Publishers, Dordrecht, The Netherlands, 2000.

[13] A. I. Brandão Lopes Pinto, F. S. de Blasi, and F. Iervolino, "Uniqueness and existence theorems for differential equations with convex valued solutions," Bollettino della Unione Matematica Italiana, vol. 4, no. 3, pp. 1-12, 1970.

[14] G. N. Galanis, T. G. Bhaskar, V. Lakshmikantham, and P. K. Palamides, "Set valued functions in Fréchet spaces: continuity, Hukuhara differentiability and applications to set differential equations," Nonlinear Analysis A, vol. 61, no. 4, pp. 559-575, 2005.

[15] V. A. Plotnikov and L. I. Plotnikova, "Averaging of equations of controlled motion in a metric space," Cybernetics and Systems Analysis, vol. 33, no. 4, pp. 601-606, 1997.

[16] B. van Cutsem, "Martingales de multiapplications à valeurs convexes compactes," Les Comptes Rendus de l'Académie des sciences, vol. 269, pp. 429-432, 1969.

[17] E. P. Avgerinos and N. S. Papageorgiou, "Almost sure convergence and decomposition of multivalued random processes," The Rocky Mountain Journal of Mathematics, vol. 29, no. 2, pp. 401-435, 1999.

[18] S. Bagchi, "On a.s. convergence of classes of multivalued asymptotic martingales," Annales de l'Institut Henri Poincaré, vol. 21, no. 4, pp. 313-321, 1985.

[19] F. Hiai and H. Umegaki, "Integrals, conditional expectations, and martingales of multivalued functions," Journal of Multivariate Analysis, vol. 7, no. 1, pp. 149-182, 1977.

[20] E. J. Jung and J. H. Kim, "On set-valued stochastic integrals," Stochastic Analysis and Applications, vol. 21, no. 2, pp. 401-418, 2003.

[21] B. K. Kim and J. H. Kim, "Stochastic integrals of set-valued processes and fuzzy processes," Journal of Mathematical Analysis and Applications, vol. 236, no. 2, pp. 480-502, 1999.

[22] S. Li and L. Guan, "Fuzzy set-valued Gaussian processes and Brownian motions," Information Sciences, vol. 177, no. 16, pp. 3251-3259, 2007.

[23] S. Li and A. Ren, "Representation theorems, set-valued and fuzzy set-valued Ito integral," Fuzzy Sets and Systems, vol. 158, no. 9, pp. 949-962, 2007.

[24] M. T. Malinowski, "On a new set-valued stochastic integral with respect to semimartingales and its applications," Journal of Mathematical Analysis and Applications, vol. 408, no. 2, pp. 669-680, 2013.

[25] N. S. Papageorgiou, "On the efficiency and optimality of allocations II," SIAM Journal on Control and Optimization, vol. 24, no. 3, pp. 452-479, 1986.

[26] B. van Cutsem, "Martingales de convexes fermés aléatoires en dimension finie," Annales de l'Institut Henri Poincaré B, vol. 8, pp. 365-385, 1972.

[27] M. T. Malinowski and M. Michta, "Stochastic set differential equations," Nonlinear Analysis A, vol. 72, no. 3-4, pp. 1247-1256, 2010. 
[28] M. Michta, "Set-valued random differential equations in Banach space," Discussiones Mathematicae Differential Inclusions, vol. 15, no. 2, pp. 124-200, 1995.

[29] M. Michta, "Continuity properties of solutions of multivalued equations with white noise perturbation," Journal of Applied Mathematics and Stochastic Analysis, vol. 10, no. 3, pp. 239-248, 1997.

[30] M. Michta, "On set-valued stochastic integrals and fuzzy stochastic equations," Fuzzy Sets and Systems, vol. 177, pp. 1-19, 2011.

[31] I. Mitoma, Y. Okazaki, and J. Zhang, "Set-valued stochastic differential equation in M-type 2 Banach space," Communications on Stochastic Analysis, vol. 4, no. 2, pp. 215-237, 2010.

[32] J. Li, S. Li, and Y. Ogura, "Strong solution of Itô type set-valued stochastic differential equation," Acta Mathematica Sinica, vol. 26, no. 9, pp. 1739-1748, 2010.

[33] N. U. Ahmed, "Nonlinear stochastic differential inclusions on Banach space," Stochastic Analysis and Applications, vol. 12, no. 1, pp. 1-10, 1994.

[34] J. P. Aubin and G. da Prato, "The viability theorem for stochastic differential inclusions," Stochastic Analysis and Applications, vol. 16, no. 1, pp. 1-15, 1998.

[35] M. Kisielewicz, "Set-valued stochastic integrals and stochastic inclusions," Stochastic Analysis and Applications, vol. 15, no. 5, pp. 783-800, 1997.

[36] M. Kisielewicz, Stochastic differential inclusions and applications, Springer, New York, NY, USA, 2013.

[37] M. Kisielewicz, M. Michta, and J. Motyl, "Set valued approach to stochastic control. Part. I and II," Dynamic Systems and Applications, vol. 12, no. 3-4, pp. 405-466, 2003.

[38] J. Krasińska and M. Michta, "A note on stochastic inclusions approach for fuzzy stochastic differential equations driven by semimartingales," Dynamic Systems and Applications, vol. 22, no. 4, pp. 503-516, 2013.

[39] M. T. Malinowski and M. Michta, "The interrelation between stochastic differential inclusions and set-valued stochastic differential equations," Journal of Mathematical Analysis and Applications, vol. 408, no. 2, pp. 733-743, 2013.

[40] M. Michta and J. Motyl, "Stochastic inclusions with a nonLipschitz right hand side," in Stochastic Differential Equations, N. Halidias, Ed., pp. 189-232, Nova Scientic, 2011.

[41] W. Y. Fei and Y. Liang, "Stochastic set differential equations driven by local martingales under the non-Lipschitzian condition," Acta Mathematica Sinica, vol. 56, no. 4, pp. 561-574, 2013.

[42] W. Y. Fei and D. F. Xia, "On solutions to stochastic set differential equations to Itô type under the non-Lipschitzian condition," Dynamic Systems and Applications, vol. 22, no. 1, pp. 137-156, 2013.

[43] W. Fei, "Regularity and stopping theorem for fuzzy martingales with continuous parameters," Information Sciences, vol. 169, no. 1-2, pp. 175-187, 2005.

[44] W. Fei, "On the theory of (dual) projection for fuzzy stochastic processes," Stochastic Analysis and Applications, vol. 23, no. 3, pp. 449-474, 2005.

[45] W. Y. Fei, "A generalization of Bihari's inequality and fuzzy random differential equations with non-Lipschitz coefficients," International Journal of Uncertainty, Fuzziness and KnowledgeBased Systems, vol. 15, no. 4, pp. 425-439, 2007.

[46] W. Y. Fei and R. Wu, "Doob's decomposition theorem for fuzzy (super) submartingales," Stochastic Analysis and Applications, vol. 22, no. 3, pp. 627-645, 2004.
[47] W. Y. Fei, R. Q. Wu, and S. Shao, "Doob's stopping theorem for fuzzy (super, sub) martingales with discrete time," Fuzzy Sets and Systems. An International Journal in Information Science and Engineering, vol. 135, no. 3, pp. 377-390, 2003.

[48] M. T. Malinowski, "Strong solutions to stochastic fuzzy differential equations of Itô type," Mathematical and Computer Modelling, vol. 55, no. 3-4, pp. 918-928, 2012.

[49] M. T. Malinowski, "Itô type stochastic fuzzy differential equations with delay," Systems and Control Letters, vol. 61, no. 6, pp. 692-701, 2012.

[50] M. T. Malinowski, "Some properties of strong solutions to stochastic fuzzy differential equations," Information Sciences, vol. 252, pp. 62-80, 2013.

[51] M. T. Malinowski, "Approximation schemes for fuzzy stochastic integral equations," Applied Mathematics and Computation, vol. 219, no. 24, pp. 11278-11290, 2013.

[52] X. R. Mao, Exponential Stability of Stochastic Differential Equations, Marcel Dekker, New York, NY, USA, 1994.

[53] B. Øksendal, Stochastic Differential Equations: An Introduction with Applications, Springer, Berlin, Germany, 6th edition, 2005.

[54] N. Ikeda and S. Watanabe, Stochastic Differential Equations and Diffusion Processes, North-Holland Publishing, Amsterdam, The Netherlands, 1981. 


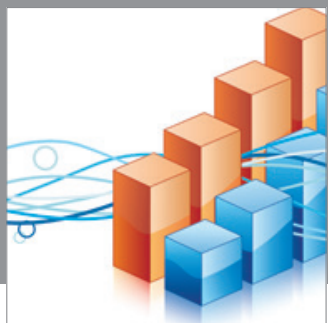

Advances in

Operations Research

mansans

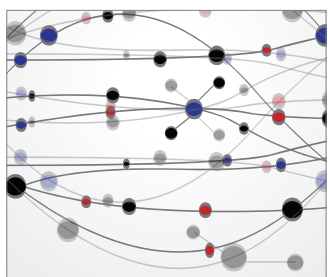

The Scientific World Journal
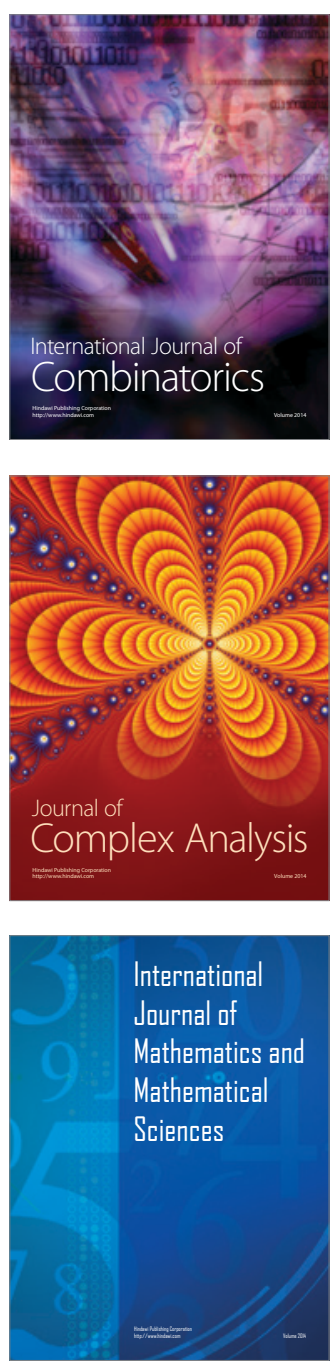
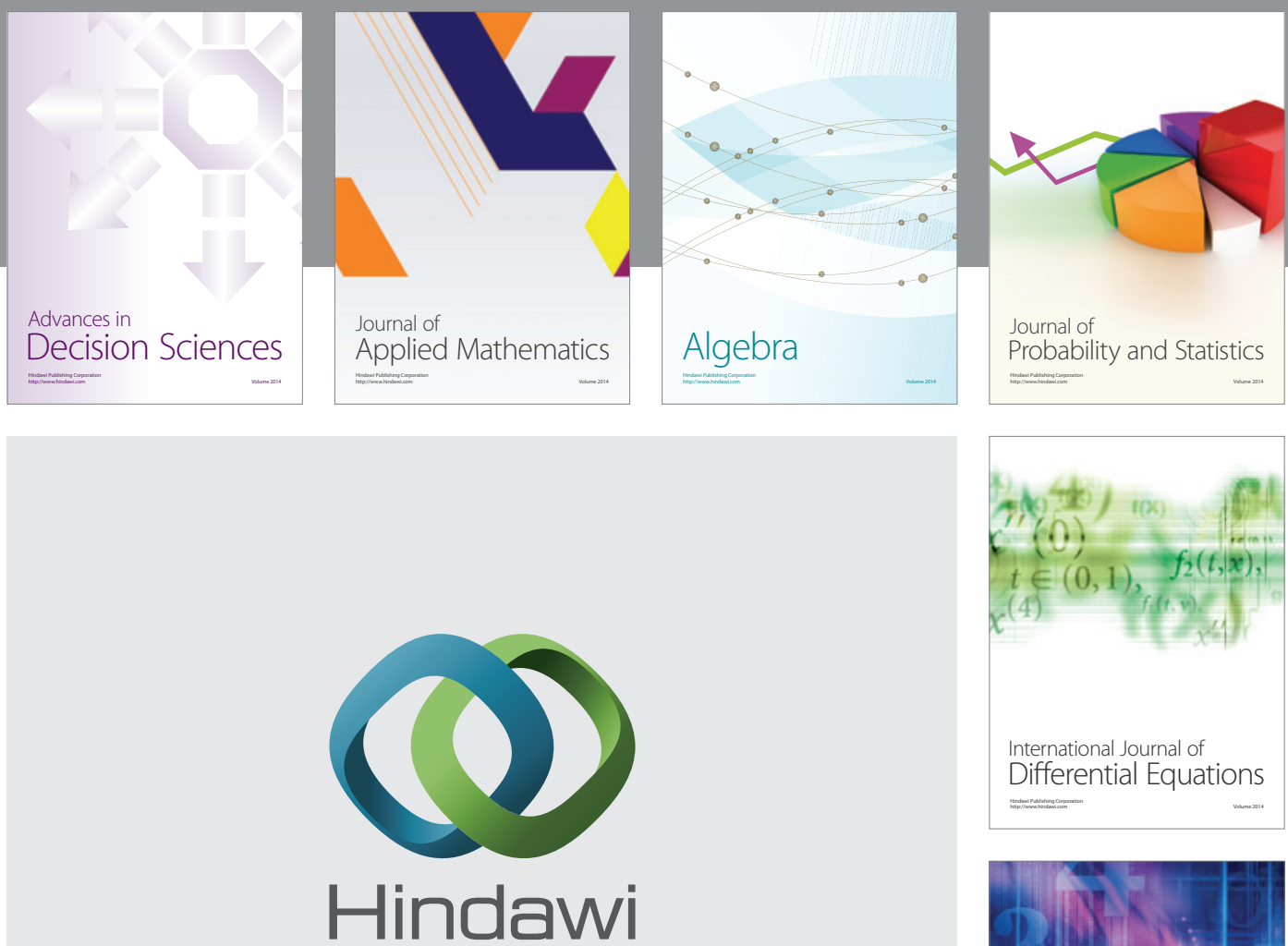

Submit your manuscripts at http://www.hindawi.com
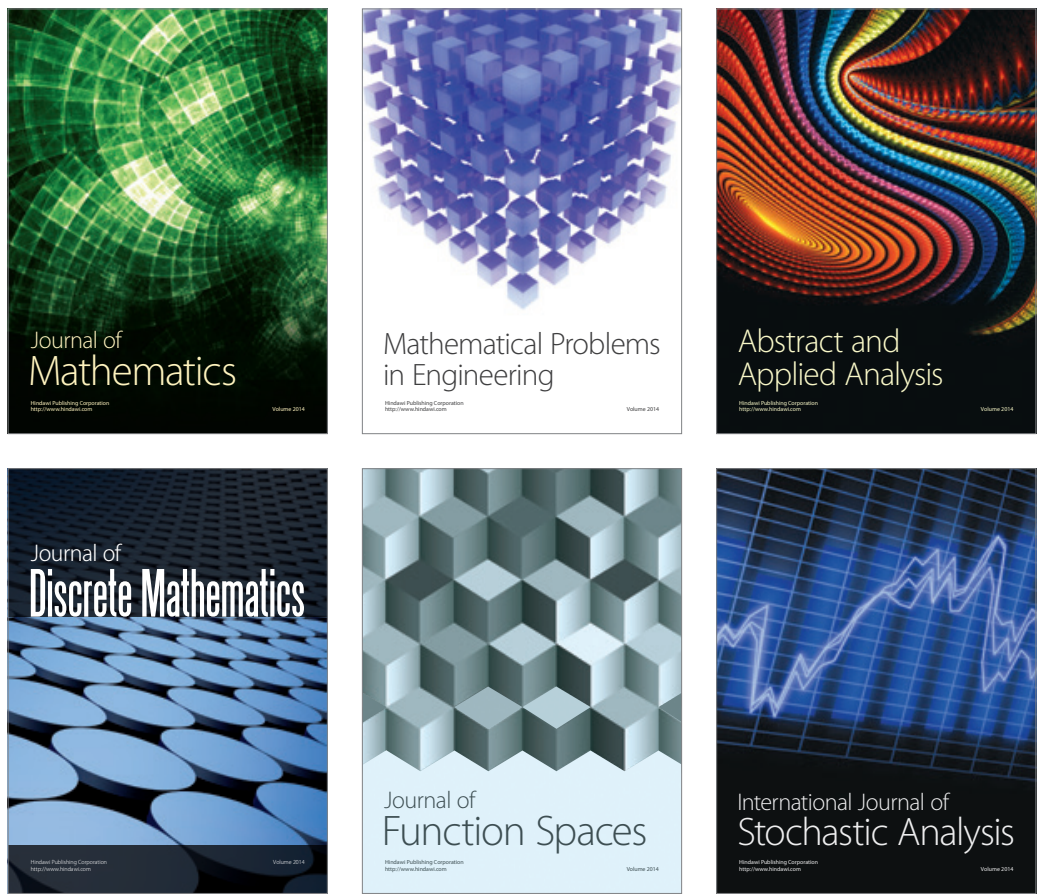

Journal of

Function Spaces

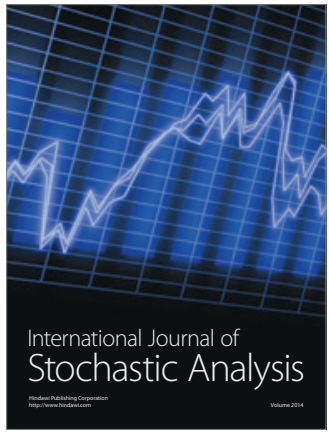

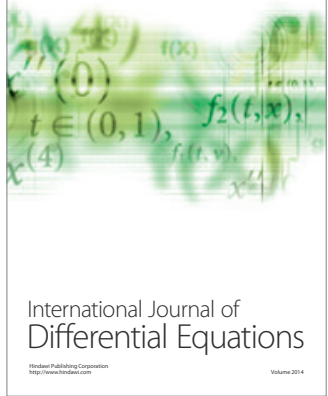
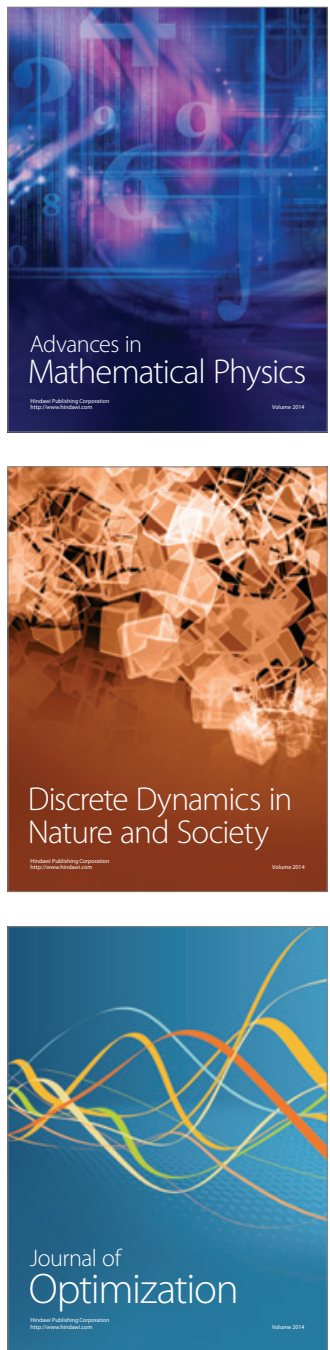